\title{
THE BANACH-SAKS PROPERTY FROM A LOCALLY SOLID VECTOR LATTICE POINT OF VIEW
}

\author{
OMID ZABETI
}

\begin{abstract}
The aim of this note is to consider different notions for the Banach-Saks property in locally solid vector lattices as an extension for the known concepts of the Banach-Saks property in Banach lattices. We investigate relations between them; in particular, we shall characterize spaces in which, these notions agree.
\end{abstract}

\section{MOTIVATION AND PRELIMINARIES}

Let us start with some motivation. Let $E$ be a Banach lattice. It is known that there are several non-equivalent notions related to the Banach-Saks property on $E$ which have many considerable connections together. In particular, we are able to characterize order continuity and also reflexivity of $E$ in terms of these relations ( see [7, 12] for more information).

Although, Banach lattices are the most important part of the category of all locally solid vector lattices, but the general theory of locally solid vector lattices has practical applications in economics, risk measures, etc ( see [2, Chapter 9]). Furthermore, the set of all examples in this category is much more than the examples in Banach lattice theory so that in this case, we have a wider range of spaces. All of these reasons, motivate us to investigate several known notions which are defined in Banach lattices in the category of all locally solid vector lattices; one principal notion is the BanachSaks property.

In this paper, we consider the Banach-Saks property ( in four non-equivalent ways) in the category of all locally solid vector lattices as an extension for the corresponding properties in the category of all Banach lattices. Recently, some different notions for the Banach-Saks property in Banach lattices have been considered in [12]. Our attempt in this note is to investigate and possibly extend the related results in that paper to the category of all locally solid vector lattices.

Date: October 27, 2020.

2020 Mathematics Subject Classification. 46A40.

Key words and phrases. Locally solid Banach-Saks property, locally solid disjoint Banach-Saks property, locally solid strong unbounded Banach-Saks property, locally solid unbounded Banach-Saks property, locally solid vector lattice. 
Now, we recall some preliminaries we need in the sequel. A vector lattice $X$ is called order complete ( $\sigma$-order complete) if every non-empty bounded above subset ( bounded above countable subset) of $X$ has a supremum. A set $S \subseteq X$ is called a solid set if $x \in X, y \in S$ and $|x| \leq|y|$ imply that $x \in S$. Also, recall that a linear topology $\tau$ on a vector lattice $X$ is referred to as locally solid if it has a local basis at zero consisting of solid sets. Suppose $X$ is a locally solid vector lattice. A net $\left(x_{\alpha}\right) \subseteq X$ is said to be unbounded convergent to $x \in X$ ( in brief, $x_{\alpha} \stackrel{u \tau}{\rightarrow} x$ ) if for each $u \in X_{+}$, $\left|x_{\alpha}-x\right| \wedge u \stackrel{\tau}{\rightarrow} 0$; suppose $\tau_{1}=\|$.$\| and \tau_{2}=$ the absolute weak topology $\left(|\sigma|\left(X, X^{\prime}\right)\right.$, in notation), we write $x_{\alpha} \stackrel{\text { un }}{\longrightarrow} x$ and $x_{\alpha} \stackrel{\text { uaw }}{\longrightarrow} x$, respectively; for more details, see [5, 8, 11]. Observe that a locally solid vector lattice $(X, \tau)$ is said to have the Levi property if every $\tau$-bounded upward directed set in $X_{+}$has a supremum. Moreover, recall that a locally solid vector lattice $(X, \tau)$ possesses the Lebesgue property if for every net $\left(u_{\alpha}\right)$ in $X, u_{\alpha} \downarrow 0$ implies that $u_{\alpha} \stackrel{\tau}{\rightarrow} 0$. Finally, observe that $(X, \tau)$ has the pre-Lebesgue property if $0 \leq u_{\alpha} \uparrow \leq u$ implies that the net $\left(u_{\alpha}\right)$ is $\tau$-Cauchy. For undefined terminology and related notions, see [1, 2, 3]. All locally solid vector lattices in this note are assumed to be Hausdorff.

\section{MAIN RESULT}

First, we present the main definition of the paper: The Banach-Saks property with a topological flavor; for recent progress in Banach lattices, see [7, 12].

Definition 1. Suppose $(X, \tau)$ is a locally solid vector lattice. $X$ is said to have

(i) The Banach-Saks property ( LSBSP, for short) if every $\tau$-bounded sequence $\left(x_{n}\right) \subseteq X$ has a subsequence $\left(x_{n_{k}}\right)$ whose Cesàro means is $\tau$-convergent; that is $\left(\frac{1}{n} \sum_{k=1}^{n} x_{n_{k}}\right)$ is $\tau$-convergent.

(ii) The strong unbounded Banach-Saks property ( LSSUBSP, in notation) if every $\tau$-bounded $u \tau$-null sequence $\left(x_{n}\right) \subseteq X$ has a subsequence $\left(x_{n_{k}}\right)$ whose Cesàro means is $\tau$-convergent.

(iii) The unbounded Banach-Saks property ( LSUBSP, in notation) if every $\tau$ bounded $u|\sigma|\left(X, X^{\prime}\right)$-null sequence $\left(x_{n}\right) \subseteq X$ has a subsequence $\left(x_{n_{k}}\right)$ whose Cesàro means is $\tau$-convergent.

(iv) The disjoint Banach-Saks property ( LSDBSP, in brief) if every $\tau$-bounded disjoint sequence $\left(x_{n}\right) \subseteq X$ has a subsequence $\left(x_{n_{k}}\right)$ whose Cesàro means is $\tau$-convergent. 
It is clear that LSBSP implies LSSUBSP, LSUBSP and LSDBSP. Now, we extend [5, Theorem 3.2] to the category of all Fréchet spaces. The proof is essentially the same; just, it is enough to replace norm with the compatible translation-invariant metric. Recall that a metrizable locally solid vector lattice $X$ is a Fréchet space if it is complete.

Lemma 2. Suppose $(X, \tau)$ is a Fréchet space and $\left(x_{\alpha}\right)$ is a u $\tau$-null net in $X$. Then there exists an increasing sequence of indices $\left(\alpha_{k}\right)$ and a disjoint sequence $\left(d_{k}\right)$ such that $x_{\alpha_{k}}-d_{k} \stackrel{\tau}{\rightarrow} 0$.

Note that metrizability is essential in Lemma 2 and can not be removed. Consider $X=\ell_{1}$ with the absolute weak topology. Put $u_{n}=(\underbrace{0, \ldots, 0}_{n-\text { times }}, \underbrace{n, \ldots, n}_{n-\text { times }}, 0, \ldots)$. It is easy to see that $u_{n} \rightarrow 0$ in the unbounded absolute weak topology ( uaw-topology); for more details, see [11]. Now, suppose $\left(d_{n}\right)$ is any disjoint sequence in $X$. It is easy to see that the sequence $\left(x_{n}-d_{n}\right)$ has at least one component with value $n$. This means that $x_{n}-d_{n} \nrightarrow 0$ in the weak topology so that in the absolute weak topology.

In this step, let us recall definition of the $A M$-property; for more details, see [10]. Suppose $(X, \tau)$ is a locally solid vector lattice. We say that $X$ has the $A M$-property provided that for every bounded set $B \subseteq X, B^{\vee}$ is also bounded with the same scalars; namely, given a zero neighborhood $V$ and any positive scalar $\alpha$ with $B \subseteq \alpha V$, we also have $B^{\vee} \subseteq \alpha V$. Note that by $B^{\vee}$, we mean the set of all finite suprema of elements of $B$. Observe that $B^{\vee}$ can be considered as an increasing net in $X$.

Lemma 3. Suppose $X$ is a locally solid vector lattice with the AM-property and $U$ is an arbitrary solid zero neighborhood in $X$. Then, for each $m \in \mathbb{N}, U \vee \ldots \vee U=U$, in which $U$ is appeared m-times.

Proof. Suppose $x \in U$; without loss of generality, we may assume that $x \geq 0$ so that $x=x \vee 0 \ldots \vee 0$. Therefore, $x \in U \vee \ldots \vee U$. For the other direction, suppose that $x_{1}, \ldots, x_{m} \in U$. Put $F=\left\{x_{1}, \ldots, x_{m}\right\} ; F$ is bounded so that by definition of the $A M$-property, $x_{1} \vee \ldots \vee x_{m} \in U$.

Lemma 4. Suppose $X$ is a locally solid vector lattice with the AM-property. Then $X$ possesses the LSDBSP.

Proof. Suppose $\left(x_{n}\right)$ is a $\tau$-bounded disjoint sequence in $X$ and $V$ is an arbitrary solid zero neighborhood in $X$. There exists a positive scalar $\gamma$ with $\left(x_{n}\right) \subseteq \gamma V$. Assume 
that $B$ is the set of all finite suprema of elements of $\left(x_{n}\right)$. By the $A M$-property, $B$ is also bounded and $B \subseteq \gamma V$. Therefore, for any subsequence $\left(x_{n_{k}}\right)$ of $\left(x_{n}\right)$, we have

$$
\frac{1}{n} \Sigma_{k=1}^{n} x_{\alpha_{k}}=\frac{1}{n} \bigvee_{k=1}^{n} x_{\alpha_{k}} \in \frac{\gamma}{n} V \subseteq V,
$$

for sufficiently large $n$. This would complete the proof.

Proposition 5. Suppose $X$ is a $\sigma$-order complete locally solid vector lattice. Then LSUBSP in $X$ implies LSDBSP.

Proof. First, assume that $X$ possesses the pre-Lebesgue property. Suppose $\left(x_{n}\right)$ is a $\tau$ bounded disjoint sequence in $X$. By [8, Theorem 4.2], $x_{n} \stackrel{u \tau}{\longrightarrow} 0$ so that $x_{n} \stackrel{u|\sigma|\left(X, X^{\prime}\right)}{\longrightarrow} 0$. Therefore, there exists an increasing sequence $\left(n_{k}\right)$ of indices such that the sequence $\left(\frac{1}{n} \sum_{k=1}^{n} x_{n_{k}}\right)$ is $\tau$-convergent. Now, suppose $X$ does not have the pre-Lebesgue property. By [2, Theorem 3.28], $\ell_{\infty}$ is lattice embeddable in $X$ so that $X$ can not have LSUBSP. This would complete the proof.

For the converse, we have the following.

Theorem 6. Suppose $(X, \tau)$ is a $\sigma$-order complete locally convex-solid vector lattice which is also a Fréchet space. Then LSDBSP results LSUBSP in $X$ if and only if $X$ does not contain a lattice copy of $\ell_{\infty}$.

Proof. The direct implication is trivial since $\ell_{\infty}$ does not have LSUBSP. For the other side, assume that $\left(x_{n}\right)$ is a $\tau$-bounded $u|\sigma|\left(X, X^{\prime}\right)$-null sequence in $X$. This means that for each $u \in X_{+}, x_{n} \wedge u \rightarrow 0$ absolutely weakly. By [2, Theorem 6.17 and Theorem 3.28], $x_{n} \wedge u \stackrel{\tau}{\rightarrow} 0$. By Lemma 2 , there exist an increasing sequence $\left(n_{k}\right)$ of indices and a disjoint sequence $\left(d_{k}\right)$ in $X$ such that $x_{n_{k}}-d_{k} \stackrel{\tau}{\rightarrow} 0$. By passing to a subsequence, we may assume that $\lim \frac{1}{m} \sum_{k=1}^{m} d_{k} \rightarrow 0$. Now, the result follows from the following inequality; observe that when a sequence in a topological vector space is null, then so is its Cesàro means.

$$
\frac{1}{m} \sum_{k=1}^{m} x_{n_{k}}-\frac{1}{m} \sum_{k=1}^{m} d_{k}=\frac{1}{m} \sum_{k=1}^{m}\left(x_{n_{k}}-d_{k}\right) \rightarrow 0 .
$$

It is clear that LSUBSP implies LSSUBSP. For the converse, we have the following characterization.

Theorem 7. Suppose $(X, \tau)$ is a $\sigma$-order complete locally convex-solid vector lattice. Then LSSUBSP in $X$ implies LSUBSP if and only if $X$ does not contain a lattice copy of $\ell_{\infty}$. 
Proof. Note that $\ell_{\infty}$ possesses LSSUBSP by [6, Theorem 2.3] but it fails to have LSUBSP; in this case, it should have the weak Banach-Saks property which is not possible. Now, suppose $X$ does not contain a lattice copy of $\ell_{\infty}$. By [2, Theorem 3.28], $X$ possesses the pre-Lebesgue property. Now, suppose $\left(x_{n}\right) \subseteq X$ is a bounded sequence which is $u|\sigma|\left(X, X^{\prime}\right)$-null. Therefore, by [2, Theorem 6.17], $\left(x_{n}\right)$ is also $u \tau$ null. By the assumption, there exists a subsequence $\left(x_{n_{k}}\right)$ of $\left(x_{n}\right)$ whose Cesàro means is convergent, as claimed.

Proposition 8. Suppose a topologically complete locally convex-solid vector lattice $(X, \tau)$ possesses LSBSP. Then $(X, \tau)$ possesses the Lebesgue and Levi properties.

Proof. Suppose not. By [9, Theorem 1], $X$ contains a lattice copy of $c_{0}$. This means that $X$ fails to have LSBSP.

Proposition 9. Suppose $(X, \tau)$ is an atomic locally solid vector lattice which possesses the Lebesgue and Levi properties. Then LSSUBSP in $X$ implies LSBSP.

Proof. Suppose $\left(x_{n}\right)$ is a $\tau$-bounded sequence in $X$. By [4, Theorem 6], there exists a subsequence $\left(x_{n_{k}}\right)$ of $\left(x_{n}\right)$ which is $u \tau$-convergent. By the assumption, there is a sequence $\left(n_{k_{k^{\prime}}}\right)$ of indices such that the sequence $\left(x_{n_{k_{k^{\prime}}}}\right)$ has a convergent Cesàro means. This would complete the proof.

Corollary 10. Suppose $(X, \tau)$ is an atomic locally solid vector lattice which possesses the Lebesgue and Levi properties. Then LSUBSP in X implies LSBSP.

Observe that atomic assumption is necessary in Proposition 9 and can not be omitted; consider $X=L^{1}[0,1]$ with the norm topology. It possesses the Lebesgue and Levi properties but it is not atomic. Suppose $\left(f_{n}\right)$ is a bounded sequence in $X$ which is un-convergent to zero; by [5, Corollary 4.2], it is convergent in measure. By [1, Theorem 1.82], there exists a subsequence $\left(f_{n_{k}}\right)$ of $\left(f_{n}\right)$ which is also pointwise convergent. By [1, Corollary 1.86], it is uniformly relatively convergent to zero. This implies that the Cesàro means of this subsequence is convergent. So, $X$ possesses LSSUBSP; nevertheless, it fails to have LSBSP, certainly.

\section{REFERENCES}

[1] Y. Abramovich and C.D. Aliprantis, An invitation to Operator theory, Vol. 50. Providence, RI: American Mathematical Society, 2002.

[2] C. D. Aliprantis and O. Burkinshaw, Locally solid Riesz spaces with applications to economics, Mathematical Surveys and Monographs, 105, American Mathematical Society, Providence, 2003.

[3] C.D. Aliprantis and O. Burkinshaw, Positive operators, Springer, 2006. 
[4] Y. Dabboorasad, E. Y. Emelyanov, and M. A. A. Marabeh, um-topology in multi-normed vector lattices, Positivity, 22(2) (2018), pp. 653-667.

[5] Y. Deng, M O'Brien, and V. G. Troitsky, Unbounded norm convergence in Banach lattices, Positivity, 21(3) (2017), pp. 963-974.

[6] M. Kandić, M. A. A. Marabeh, and V. G. Troitsky, Unbounded norm topology in Banach lattices, J. Math. Anal. Appl., 451 (1)(2017), pp. 259-279.

[7] N. Gao, V. G. Troitsky, and F. Xanthos, Uo-convergence and its applications to Cesàro means in Banach lattices, Israel J. Math, 220 (2017), pp. 649-689.

[8] M. A. Taylor, Unbounded topologies and uo-convegence in locally solid vector lattices, J. Math. Anal. Appl., 472 (1)(2019), pp. 981-1000.

[9] W. Wnuk, Locally solid Riesz spaces not containing $c_{0}$, Bull. Polish Acad. Sci. Math., 36(1-2), pp. 51--56. (1988)

[10] O. Zabeti, AM-Spaces from a locally solid vector lattice point of view with applications, Bulletin. Iran. Math. Society, to appear, doi: 10.1007/s41980-020-00458-7.

[11] O. Zabeti, Unbounded absolute weak convergence in Banach lattices, Positivity, 22(1) (2018), pp. 501-505.

[12] O. Zabeti, Unbounded continuous operators and unbounded Banach-Saks property in Banach lattices, Preprint, arXiv: 2007.05734v3 [math.FA].

(O. Zabeti) Department of Mathematics, Faculty of Mathematics, Statistics, and Computer science, University of Sistan and Baluchestan, Zahedan, P.O. Box 98135674. IRAN

Email address: o.zabeti@gmail.com 